\title{
A TECHNIQUE FOR PREPARING A CAST IN SYNTHETIC RESIN OF THE CAVITIES AND BLOOD VESSELS OF THE HEART
}

\author{
BY \\ D. H. TOMPSETT \\ From the Anatomy Department of the Royal College of Surgeons of England, London
}

(RECEIVED FOR PUBLICATION NOVEMBER 18, 1953)

This technique provides a method for demonstrating the principal branches of the coronary arteries, and the larger cardiac veins. At the same time a cast of the cavities of the heart is produced. Although a relatively low injection pressure is used, the thin-walled cavities of the atria and the right ventricle are considerably distended, so that the cast of these cavities is larger than the cavities were themselves in life. But, in spite of this defect, the cast of the cavities serves the useful purpose of making it easy to visualize the form of the heart which contained them, and in this way increases the value of the cast of the vessels.

\section{MATERIAL}

A fresh post-mortem heart, to which the lungs and ascending aorta are attached, is required for this technique.

\section{METHOD}

Preparation of Material for Injection.-The lungs are removed carefully so that about an inch of each of the four pulmonary veins remains attached to the left atrium. Short pieces of flanged glass tubing are tied into the two left pulmonary veins, the left branch of the pulmonary artery, the superior and inferior venae cavae, and the aorta. The rest of the vessels are ligatured. Fig. 1 shows a diagram of the heart with these tubes attached.

The first step is to wash out the blood from both the cavities and the vessels of the heart as completely as possible. If any blood remains in the vessels during fixation of the heart it tends to collect into clots which will obstruct the flow of the resin and result in an incomplete cast. The washing out process is begun with $2 \%$ sodium citrate and completed with tap water. Citrate is poured from a funnel held about a foot above the heart into the left atrium via one of the pulmonary veins. At first it is allowed to escape via the other unligatured pulmonary vein. When most of the blood in the left atrium has been removed, the escape from the latter is closed by means of a short piece of rubber tubing with a screw clamp, fixed to the glass tube in the pulmonary vein, so that the citrate flows into the left ventricle, escaping via the aorta. Finally the opening from the cut end of the aorta is closed so that the citrate flows only into the coronary arteries, escaping via the coronary veins. Then the right side of the heart is washed out in a similar way.

Next the cold tap is connected to the left atrium and turned on fairly full with the two escape holes open. The escape holes are partially closed by means of the screw clamps attached to them, so that a sufficient pressure of water is developed to cause some of the water to flow into the coronary arteries, but without causing excessive distension of the atrium. After about an hour the right side of the heart is treated in a similar way.

Finally the heart is impregnated with $70 \%$ spirit via the coronary arteries, and then the heart is placed in $70 \%$ spirit for four days. After the first 24 hours

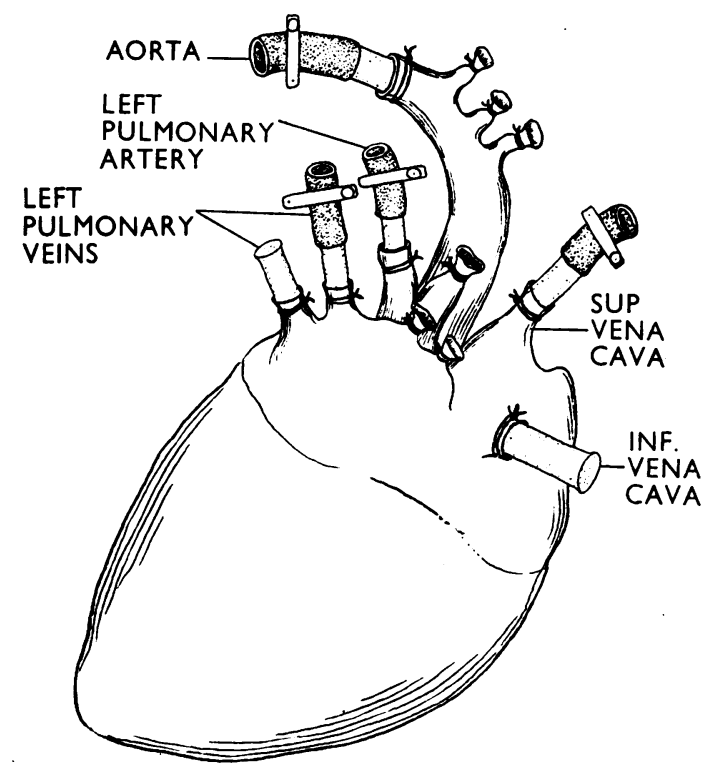

FIG. 1. - The heart with six glass tubes attached. Four of these tubes are used as escape tubes, their openings being controlled by rubber tubing and screw clamps. The other two are for injection of the resin. 
the left side of the heart is flushed with spirit to dislodge and disintegrate any blood still remaining in the coronary arteries.

INJECTION OF THE RESIN.-The heart is immersed in water, and water is allowed to flow rapidly through each side of it for two hours to remove the spirit with which it is impregnated. Then it is carefully manipulated to squeeze out any air which may have entered the vessels.

The injection of the right side is made via the inferior vena cava, and of the left via one of the pulmonary veins. The resin is injected by gravity flow from glass funnels held about 6 inches above the heart. The heart is placed in a bowl of water which is maintained at about $35^{\circ} \mathrm{C}$. until the injection has been completed. The position of the funnels is adjusted so that the heart is suspended in the water, to avoid any pressure on its surface which might retard the flow of resin into the vessels.

When the apparatus has been arranged, the clamps attached to the four escape tubes are closed, and each of the funnels is filled with water to test for leaks. There is bound to be some flow of water from the funnels, as water diffuses through the tissue of the heart, but if there is a rapid flow of water this indicates a leak which must be located and closed.

Next the escape holes are opened slightly by slackening the screw clamps, and clamps are also used to close the rubber tubing connecting the funnels to the heart. These clamps are fixed slightly under water so that no air is trapped below them. It does not matter if there is a little water above these clamps, as, when the funnels are filled with resin, this water $\stackrel{\overrightarrow{\bar{c}}}{\vec{\theta}}$ will float to the surface of the resin.

Two lots of $500 \mathrm{ml}$. of the following resin mixture 듬 are prepared :

Marco resin $26 \mathrm{C} \quad \ldots \quad \ldots \quad \ldots 100 \mathrm{~g}$

$\begin{array}{lllllll}\text { Monomer C } & \ldots & \ldots & \ldots & \ldots & 15 & \mathrm{~g} .\end{array}$

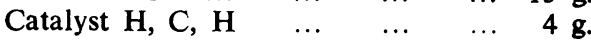

Marco pigment crimson lake (M 23)

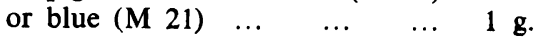

$\begin{array}{llllll}\text { Accelerator } \mathrm{E} & \ldots & \ldots & \ldots & 3 \mathrm{ml} . *\end{array}$

The catalyst is dissolved in the monomer, aided by gentle warming, and the pigment is added. Then the $x$ monomer is incorporated with the resin. The acceler- 6 ator is added immediately before use and stirred in $N$ thoroughly.

At $20^{\circ} \mathrm{C}$. this resin mixture has a working life of about 20 minutes after the accelerator has been 을 added, but the exact setting time must be established by making a careful test with one unit of the above $c$ mixture (i.e., $100+15+4+1)=120$ g. $+3 \mathrm{ml}$. accelerator, as the setting time varies according to the room temperature and the age of the materials.

The two funnels are filled with resin. Fig. 2 shows $\oplus$ a diagram of the apparatus just before the injection 0 of the resin which is begun not more than 10 minutes before the resin is due to set. The resin is run first into the left side of the heart. The openings of the two escape tubes are held up vertically, and as soon as the resin begins to escape from them they are $\varnothing$ closed. The same procedure is applied to the right $\overrightarrow{\vec{B}}$ side. Then all the escape tubes are held upright

* These substances can be obtained from Scott Bader, Ltd., 109, Kingsway, London, W.C.2.

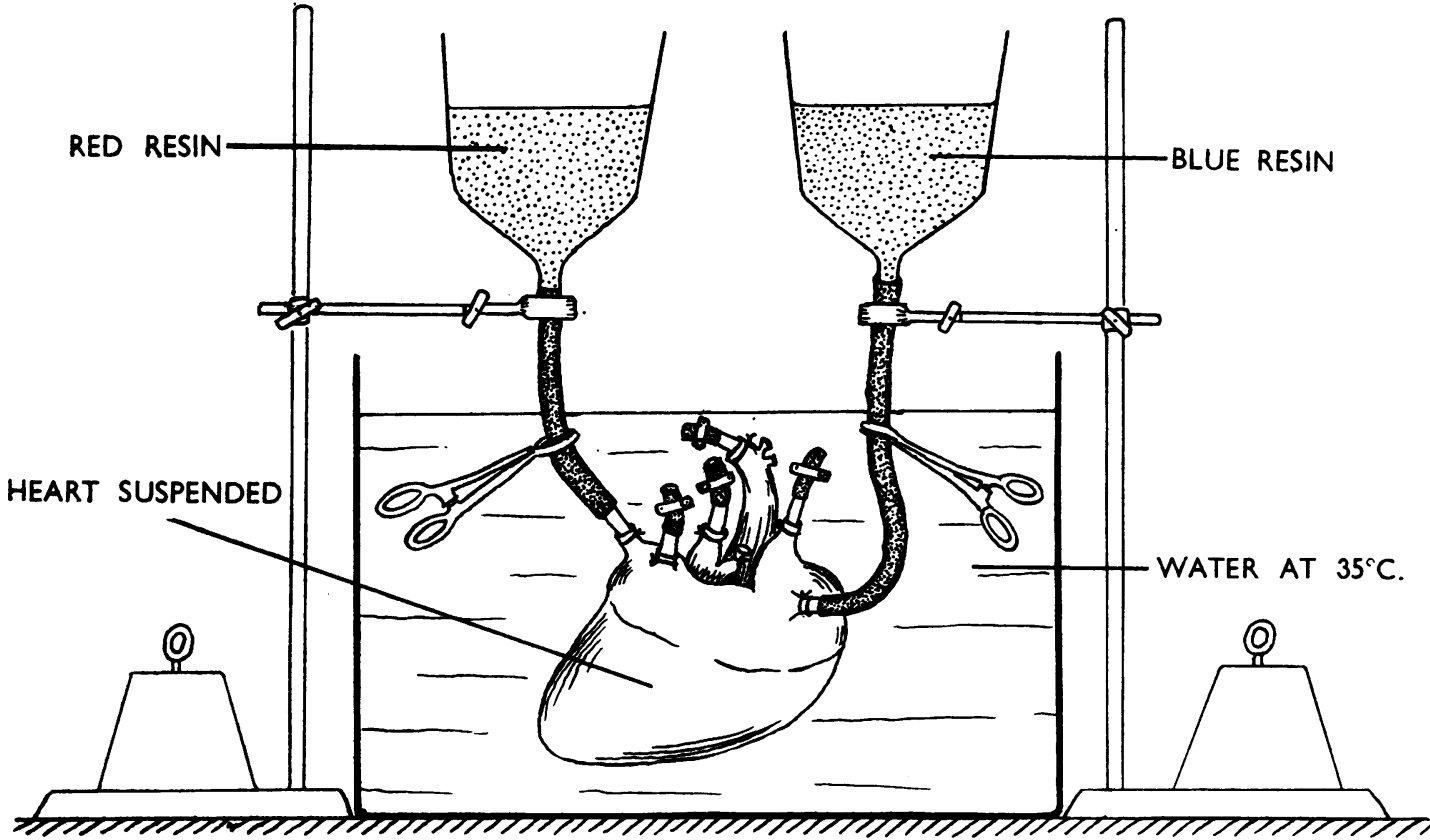

Fig. 2.-Apparatus for injection of heart with resin. 
for about a minute to allow any water still trapped in the cavities of the heart to float to their openings. These tubes are then slightly opened one by one to allow this water to escape, and closed again.

If there is any extensive leakage of resin it is controlled by applying artery forceps. The resin in the funnels provides sufficient injection pressure to displace the water in the vessels, but the vessels take some time to fill, so the resin must be allowed to flow freely until it sets.

Until the resin sets the four escape tubes (with their openings closed) are held vertically so that any water still in the cavities of the heart will float into them. As soon as the resin in the heart is sufficiently solid for there to be no flow when one of the clamps closing an escape hole is opened, the rubber tubing and the resin it contains is cut through close to all the pieces of glass tubing. This must be done before the resin has become really hard.

The heart is placed in cold water for an hour after the resin solidifies to prevent it from overheating as polymerization proceeds. After this period it is left in a water bath at about $60^{\circ} \mathrm{C}$. for two hours to accelerate the setting of the resin in the vessels.

Preliminary Treatment before Maceration.The heart must be handled very carefully after injection to avoid breaking the resin in the vessels, as, if the resin in one of the vessels is broken, when the flesh is dissolved away the cast of the broken vessel will drop off. Twenty-four hours after the injection the heart is placed on a large wad of wet cottonwool, and the glass tubes are cracked and the pieces removed. Then the projecting stumps of resin are sawn off with a hack-saw.

It is necessary to fix the casts of the left and right sides together before the heart is macerated, as otherwise the two pieces of the cast would fall apart. This is done by first exposing part of the cast of the aorta and the adjacent parts of the casts of the superior vena cava and pulmonary artery, by dissecting away the walls of these vessels. Small pieces of transparent resin are shaped by filing, so that they fit into the gaps between the aorta and the superior vena cava, and the aorta and the pulmonary artery. These pieces of resin are cemented in place with a little Marco resin $28 \mathrm{C}$ to which $10 \%$ catalyst paste $\mathrm{H}$ and $10 \%$ accelerator $\mathrm{E}$ have been added. This cement is used just before it sets. The imminence of setting is indicated by greatly increased viscosity. The above mixture sets in about 15 minutes, but several hours elapse before it becomes really hard. Another small piece of resin is cemented to the underside of the cast of the aorta, at its end, so that it forms a hook by which the heart can be suspended during maceration. A hole is drilled through the base of the cast of the aorta into which a metal rod is to be fixed later for mounting the cast.

The surface of the heart is gently palpated to discover if any of the casts of the vessels have been broken. If they have, the broken ends are repaired with resin cement, after stripping away the vessel wall. Water tends to collect in the coronary sinus, so the sinus is opened, and if it is found to be incompletely filled with resin the cavity is topped up with blue resin.

MACERATION.-A suitable container, which may be made of glass or perspex, is covered with a perspex lid, from the centre of which a piece of perspex projects downwards, with a hole drilled through it, by means of which the specimen can be suspended.

The specimen is left for at least a week in a bowl of water while the resin matures before maceration is begun. It is then suspended in concentrated hydrochloric acid for three days. After this period it is suspended in cold water, and a jet of cold water is directed on to the macerated flesh to wash it away. If during the washing it is observed that any of the casts of the vessel are broken, these must be repaired before all the macerated flesh which supports them has been washed away, or else be removed to be cemented on later. After washing has been completed, the cast of the vessels is pruned to remove resin which has not completely hardened, owing to the effect of water inhibition (see discussion). Then the cast is immersed for a few minutes in dilute ammonia to neutralize any acid still adhering in crevices.

A whitish deposit which partly covers the surface, and which is composed of incompletely set resin which has been attacked by the acid, is removed as completely as possible, first by scraping, and then by repeatedly immersing the cast in $70 \%$ spirit for one minute and allowing it to dry. The auricle of the left atrium is sawn off to display the origin of the left coronary artery.

Mounting.-One end of a piece of steel rod is threaded, and a small brass nut is screwed to the end of the thread. Then the threaded end is bent to a right angle, and the bent end is passed into the hole in the cast of the aorta and cemented with resin. Owing to the weight of these casts it is best to allow them to hang freely, rather than to attempt to mount them in some special position.

After the rod has been cemented it is left for 24 hours for the resin cement to harden. Then the cast of the aorta is sawn off as short as required, and the whole cast is sprayed with the following resin mixture, to bring out the original colours of the resin :

$$
\begin{array}{llllrl}
\text { Marco resin } 28 \mathrm{C} & \ldots & \ldots & \ldots & 50 \mathrm{~g} . \\
\text { Monomer C } \ldots & \ldots & \ldots & \ldots & 20 \mathrm{~g} . \\
\text { Catalyst H, C, } \mathrm{H} & \ldots & \ldots & \ldots & 1 \mathrm{~g} \\
\text { Acetone } & \ldots & \ldots & \ldots & \ldots & 10 \mathrm{~g} . \\
\text { Accelerator E } & \ldots & \ldots & \ldots & 2 \mathrm{ml} .
\end{array}
$$

After spraying, the cast must be protected from dust, as the surface remains slightly tacky for some time.

A piece of copper tubing into which the steel rod will just fit is fixed vertically in a wooden base, and, after sliding the steel rod to which the cast is attached into it, the brass nut is soldered to the copper tube. If the steel rod is soldered directly to the copper tube, 
the resulting joint is not secure. Finally, the cast is covered with a perspex dust cover. Fig. 3 shows a photograph of a cast from behind. In this view the coronary sinus is seen receiving the left marginal vein and the inferior interventricular (middle cardiac) vein. The right marginal vein is seen with its
BRITTLENESS OF RESIN.-Although the resin sets $\overrightarrow{\vec{s}}$ into a hard substance, relatively fine filaments of $i \mathbb{C}^{\circ}$ are rather brittle. When the resin is wet it is much음 more flexible than when dry, and so the brittleness is reduced, but nevertheless very great care must

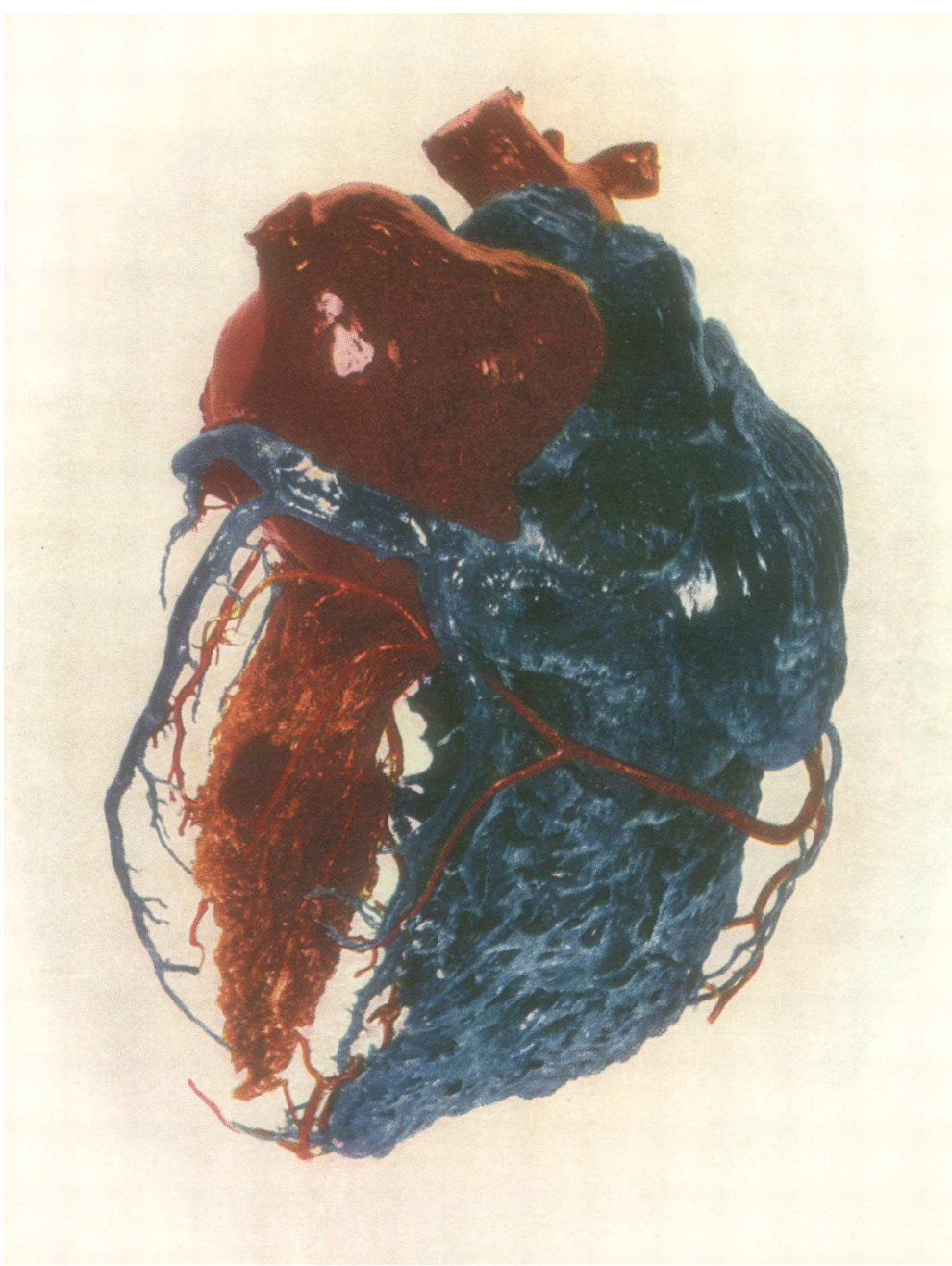

FIG. 3.-Photograph of cast of human heart from behind $\left(\times \frac{2}{3}\right)$.

companion artery at the right side of the heart; the vein opens independently into the right atrium in this specimen.

\section{Discussion}

When synthetic resin is used to make a cast of the cavities and vessels of the heart, three physical properties of the resin must be taken into account. be taken whenever the injected heart is handled ${ }^{\circ}$ or placed anywhere, to avoid breaking the cast $\overrightarrow{\mathbb{D}}$ of the vessels.

WATER INHIBITION OF RESIN.-The setting of $\frac{\varrho}{\sigma}$ the resin is inhibited by contact with water, so that the water in the walls of the blood vessels inhibitso the setting of the surface of the resin injected into 
them. If this inhibition extends to a depth equal to the radius of the vessel, a solid cast will never be produced. Consequently it is impossible by this technique to obtain a cast of the very fine vessels. The depth to which water inhibition extends can be reduced by using a resin mixture with a relatively large catalyst and accelerator content (a rapid-setting mixture), and injecting it as short a time as is practicable before it sets, thus reducing the time during which the water is in contact with the unset resin.

Exothermic Polymerization of Resin.-The amount of catalyst and accelerator which can bz added to the resin used for the injection of hearts is limited by the fact that during the setting process a considerable amount of heat is evolved. In the case of a relatively large volume of resin, such as is required to fill the cavities of the heart, if a very rapidly setting mixture is used, this heat is evolved so rapidly that it is not possible to cool the resin effectively enough to prevent it from overheating and cracking. If a rather small heart was being injected, $1 \%$ more catalyst and accelerator than recommended above could be used, as there would be less risk of the resin in the cavities of a small heart overheating than in a large one. The increase in the catalyst and accelerator content will produce a more complete cast of the vessels, by reducing the extent of water inhibition.

\section{SUMmaRY}

A technique is described for preparing a cast of the cavities and blood vessels of the heart in synthetic resin. The blood is removed, and the heart is fixed in spirit. After filling the cavities with resin, the original heart is dissolved in acid.

I am grateful to Messrs. Bayer Products, Ltd., for lending the block of Fig. 3.

\section{A P P E N D I X}

Marco resin, monomer, and accelerator should be stored in a refrigerator.

Sunlight acts as a powerful accelerator, so care must be taken to prevent sunlight from falling on the resin mixture while it is being used for injection.

Glassware contaminated with resin is cleaned by immersing it in boiling water for an hour after the resin has set. The resin can then be easily removed.

A thin film of unset resin is removed from the hands, instruments, and glassware with swabs of cotton-wool soaked in acetone.

When spraying the cast with the resin mixture a good quality spray must be used, and the rubber bulb must be squeezed with some violence, owing to the viscosity of the mixture. This labour can be avoided by connecting a cylinder of compressed air or carbon dioxide to the spray. The spraying is controlled by arranging an escape hole in the rubber tubing which connects the compressed air to the spray, by means of a $T$ piece of glass. The air is turned slightly on, and when the spray is required the escape hole is closed with a finger. The spray must be very thoroughly washed out with acetone before the resin in it sets, as the resin cannot be dissolved afterwards.

Resin can be expelled from rubber tubing after it has set by running hot water from the tap through the tubing. 